\title{
Temperature dependence of persistent spin currents in a spin-orbit-coupled electron gas: A density-matrix approach
}

\author{
K. Bencheikh ${ }^{1}$ and G. Vignale ${ }^{2}$ \\ ${ }^{1}$ Département de Physique, Laboratoire de Physique Quantique et Systèmes Dynamiques, Université de Sétif, Setif 19000, Algeria \\ ${ }^{2}$ Department of Physics and Astronomy, University of Missouri, Columbia, Missouri 65211, USA \\ (Received 21 February 2007; revised manuscript received 10 February 2008; published 14 April 2008)
}

\begin{abstract}
We present a simple analytical method, based on the canonical density matrix, for the calculation of the equilibrium spin current as a function of temperature in a two-dimensional electron gas with both Rashba and Dresselhaus spin-orbit coupling terms. We find that the persistent spin current is extremely robust against thermal disorder: its variation with temperature is exponentially small $\left(\propto e^{-T_{F} / T}\right)$ at temperatures much smaller than the Fermi temperature $T_{F}$ and changes to a power law $T_{F} / T$ for $T \gg T_{F}$.
\end{abstract}

DOI: 10.1103/PhysRevB.77.155315 PACS number(s): 73.21.Hb, 72.25. - b, 75.47. $-\mathrm{m}$, 31.15.xg

\section{INTRODUCTION}

Spintronics is a new subfield of condensed matter physics, ${ }^{1-3}$ whose aim is to involve the spin of the electron in the operation of electronic devices. While several metalbased spintronic devices (e.g., magnetic read heads for hard disk drives and magnetic tunnel junctions for magnetic random access memories) have already been described and commercialized, the quest is still on for semiconductor-based devices, which might one day lead to the integration of logical and memory functions on the same chip.

In the past few years, the efforts of the semiconductorspintronics community have focused on the coupling of the spin and orbital degrees of freedom-the spin-orbit coupling - as means to generate and control spin-polarized currents in the absence of magnetic field. In fact, the strength of the spin orbit interaction in a semiconductor can be to a certain extent controlled by electrical means (gates). ${ }^{4,5}$ One of the first spintronic devices ever proposed, i.e., the DattaDas spin transistor, ${ }^{4}$ made use precisely of a gate-controlled Rashba spin-orbit coupling to control the flow of an ordinary electrical current in a two-dimensional electron gas. The recently discovered spin Hall effect, ${ }^{6-12}$ whereby an electrical current drives a transverse spin current flow, causing a spin accumulation at the edges of a two-dimensional electron gas, offers another way to manipulate the spin via spin-orbit coupling.

However, when it comes to spin currents, one must be very careful to distinguish between persistent currents, which can exist even in equilibrium, and genuine transport currents, which arise in response to external fields. Macroscopic spin currents are generally present in spin-orbit coupled systems at equilibrium when the total spin is not conserved. In a stationary state, the spin precession caused by spin-orbit torques must be compensated by a steady spin current so that the spin density may remain constant in time. Notice that the spin current is invariant under time reversal, so it can exist in the absence of a magnetic field.

In a recent couple of papers, Sonin ${ }^{13,14}$ examined the possibility of observing equilibrium spin currents in a controlled experiment and concluded that such currents can indeed be observed through the mechanical torque they exert on a neighboring medium. This provides additional motivation for pursuing a quantitative study of equilibrium spin currents.

In the present paper, we shall be concerned with the persistent spin current in a two-dimensional electron gas with Rashba and Dresselhaus spin-orbit interactions. For that, we consider $N$ noninteracting electrons that are confined in twodimensional electron gas (2DEG) and we assume that the single particle Hamiltonian, which includes both Rashba ${ }^{15}$ and Dresselhauss (linear) type ${ }^{16,17}$ spin-orbit couplings, is given by

$$
\hat{H}=\frac{P_{x}^{2}+P_{y}^{2}}{2 m}+\frac{\kappa}{\hbar}\left(P_{y} \sigma_{x}-P_{x} \sigma_{y}\right)+\frac{\gamma}{\hbar}\left(P_{y} \sigma_{y}-P_{x} \sigma_{x}\right),
$$

where $\kappa$ and $\gamma$ are, respectively, the Rashba and Dresselhauss coupling constants in a 2DEG.

This system has received tremendous attention in the past few years. The fact that it supports equilibrium spin currents has been first recognized by Rashba ${ }^{18}$ in the context of the debate on the nature of the spin Hall effect. Although these currents are very small (proportional to $\kappa^{3}$, where $\kappa$ is the Rashba spin-orbit coupling constant), they are conceptually important in the analysis of spin-transport phenomena. In this paper, we develop a simple density matrix formalism for the calculation of the spin current, and then apply it to the study of the temperature dependence of the spin current in the Rashba-Dresselhaus model. In view of the fact that the spin-orbit-induced splitting of the band structure is very small compared to the Fermi energy, one could have expected the spin current to decay to zero with increasing temperature on a comparably small energy scale. We will see that it is not so. Because the relevant part of the spin current operator has no matrix elements between the Rashba and/or Dresselhaus split bands, we find that the net current is the difference of contributions associated with each band and therefore the scale of its temperature dependence is given by the Fermi energy. Indeed, at low temperature, we find that the corrections to the zero-temperature results are exponentially small ( $\propto e^{-T_{F} / T}$, where $T_{F}$ is the Fermi temperature), and they decay as a power of the temperature at temperatures larger than $T_{F}$. Thus, the persistent spin current is an extremely robust property of these systems.

One may ask why use the powerful density matrix formalism rather than the usual textbook methods, in which the spin 
current is calculated from one-electron wave functions. The reason is that (i) the density matrix formalism is of course more readily generalizable to interacting systems and (ii) a most recent trend in electronic structure theory is to formulate the problem as an optimization of the energy regarded as a functional of the density matrix - the so called densitymatrix functional theory ${ }^{19}$ - thus completely bypassing the one-electron wave functions. From this point of view, the approximate density matrix of a reference system obtained by direct solution of the Bloch equation becomes the starting point for the more sophisticated density-matrix functional treatment.

This paper is organized as follows. In Sec. II, we describe the Bloch equation method for directly calculating the density matrix, which determines the equilibrium spin current. In Sec. III, we apply the general method to a very simple case and derive an analytical expression for the spin current at zero temperature. The generalization of the formalism to finite temperature and the results for the Rashba-Dresselhaus model are presented in Sec. IV. Finally, the summary and the outlook end the paper.

\section{SPIN DENSITY-MATRIX METHOD AT ZERO TEMPERATURE}

In the study on properties of the noninteracting fermions in a one particle potential, the canonical or Bloch density matrix defined, here in a joint space of spin and orbital degrees of freedom, as

$$
C_{\beta}\left(\vec{r}, \sigma ; \vec{r}^{\prime}, \sigma^{\prime}\right):=\left\langle\vec{r}, \sigma|\hat{C}| \vec{r}^{\prime}, \sigma^{\prime}\right\rangle=\left\langle\vec{r}, \sigma|\exp (-\beta \hat{H})| \vec{r}^{\prime}, \sigma^{\prime}\right\rangle
$$

is of great importance, as we will show below. Here $\sigma$ and $\sigma^{\prime}$ are the spin indices, $\beta$ is to be interpreted as mathematical variable which in general is taken to be complex and not the inverse temperature, and $\hat{C}_{\beta}=\exp (-\beta \hat{H})$ is the Bloch density operator, which satisfies the Bloch equation,

$$
-\frac{\partial \hat{C}_{\beta}}{\partial \beta}=\hat{H} \hat{C}_{\beta}=\hat{C}_{\beta} \hat{H},
$$

with

$$
\hat{H}=\frac{\vec{P}^{2}}{2 m}+\hat{V}=\sum_{i} \epsilon_{i}\left|\Psi_{i}\right\rangle\left\langle\Psi_{i}\right|,
$$

where $\left|\Psi_{i}\right\rangle$ are the eigenstates of the Schrödinger equation $\hat{H}\left|\Psi_{i}\right\rangle=\varepsilon_{i}\left|\Psi_{i}\right\rangle$, and $\hat{V}$ is a spin dependent potential operator of the form

$$
\hat{V}=V(\vec{R})+\hbar \vec{b}_{0}(\vec{R}) \cdot \vec{\sigma}+\hbar \vec{b}_{1}(\vec{R}, \vec{P}) \cdot \vec{\sigma} .
$$

Here, $V(\vec{R})$ is a scalar confining potential, the second term is a Zeeman-like term, and the last one represents the spin orbit coupling.

The Bloch density matrix is of particular interest since its knowledge enables the Dirac density matrix $\rho\left(\vec{r}, \sigma ; \vec{r}^{\prime}, \sigma^{\prime}\right)$ to be found, through the inverse Laplace transform. ${ }^{20,21}$ In fact, at $T=0$, the single particle density operator, $\hat{\rho}_{E_{F}}$ $=\sum_{\text {all } i}\left|\Psi_{i}\right\rangle\left\langle\Psi_{i}\right| \theta\left(E_{F}-\epsilon_{i}\right)$ can be rewritten, for a given fermi energy $E_{F}$, as

$$
\hat{\rho}_{E_{F}}=\frac{1}{2 \pi i} \int_{c-i \infty}^{c+i \infty} d \beta e^{\beta E_{F}} \frac{\hat{C}_{\beta}}{\beta},
$$

where we have used

$$
\theta\left(E_{F}-\epsilon_{i}\right)=\frac{1}{2 \pi i} \int_{c-i \infty}^{c+i \infty} d \beta \frac{e^{\beta\left(E_{F}-\epsilon_{i}\right)}}{\beta},
$$

with $c$ any positive constant.

Let us now consider systems of noninteracting fermions with spin $s=1 / 2$, thus we can expand the density matrix $\hat{\rho}\left(\vec{r}, \vec{r}^{\prime}\right)=\left\langle\vec{r}\left|\hat{\rho}_{E_{F}}\right| \vec{r}^{\prime}\right\rangle$ over the complete set of Pauli matrices as

$$
\hat{\rho}\left(\vec{r}, \vec{r}^{\prime}\right)=\frac{1}{2}\left[\rho\left(\vec{r}, \vec{r}^{\prime}\right) \hat{I}+\vec{\rho}\left(\vec{r}, \vec{r}^{\prime}\right) \cdot \vec{\sigma}\right] .
$$

Besides the scalar density $\rho\left(\vec{r}, \vec{r}^{\prime}\right)=\Sigma_{\sigma}\langle\sigma|\hat{\rho}| \sigma\rangle$, one also defines the spin vector density $\vec{\rho}\left(\vec{r}, \vec{r}^{\prime}\right)=\Sigma_{\sigma}\langle\sigma|\hat{\rho} \vec{\sigma}| \sigma\rangle$, where $\hat{I}$ is the unit $2 \times 2$ matrix. Now, invoking Eq. (6), we can write

$$
\begin{gathered}
\rho\left(\vec{r}, \vec{r}^{\prime}\right)=\frac{1}{2 \pi i} \int_{c-i \infty}^{c+i \infty} d \beta \frac{e^{\beta E_{F}}}{\beta}\left[\sum_{\sigma} C_{\beta}\left(\vec{r}, \sigma ; \vec{r}^{\prime}, \sigma\right)\right], \\
\vec{\rho}\left(\vec{r}, \vec{r}^{\prime}\right)=\frac{1}{2 \pi i} \int_{c-i \infty}^{c+i \infty} d \beta \frac{e^{\beta E_{F}}}{\beta}\left[\sum_{\sigma}\left\langle\vec{r}, \sigma\left|\hat{C}_{\beta} \vec{\sigma}\right| \vec{r}^{\prime}, \sigma\right\rangle\right] \\
=\frac{1}{2 \pi i} \int_{c-i \infty}^{c+i \infty} d \beta \frac{e^{\beta E_{F}}}{\beta}\left[\sum_{\sigma, \sigma^{\prime}} C_{\beta}\left(\vec{r}, \sigma ; \vec{r}^{\prime}, \sigma^{\prime}\right)\left\langle\sigma^{\prime}|\vec{\sigma}| \sigma\right\rangle\right] .
\end{gathered}
$$

In addition to the density matrix $\hat{\rho}\left(\vec{r}, \vec{r}^{\prime}\right)$, we shall also define the so-called kinetic current density matrix $\overrightarrow{\hat{j}} k i n\left(\vec{r}, \vec{r}^{\prime}\right)$ $=\frac{\hbar}{2 m i}\left(\vec{\nabla}_{\vec{r}}-\vec{\nabla}_{\vec{r}^{\prime}}\right) \hat{\rho}\left(\vec{r}, \vec{r}^{\prime}\right)$, which is similar to Eq. (8), can be decomposed in the form

$$
\overrightarrow{\hat{j}}^{k i n}\left(\vec{r}, \vec{r}^{\prime}\right)=\frac{1}{2}\left(\overrightarrow{j^{k i n}}\left(\vec{r}, \vec{r}^{\prime}\right) \hat{I}+\sum_{\lambda=1}^{3} \vec{J}_{\lambda}^{k i n}\left(\vec{r}, \vec{r}^{\prime}\right) \sigma_{\lambda}\right) .
$$

Here, $\vec{j}^{k i n}\left(\vec{r}, \vec{r}^{\prime}\right)$ is the usual quantum mechanical current density, and the set of vectors $\left(\vec{J}_{1}^{k i n}, \vec{J}_{2}^{k i n}, \vec{J}_{3}^{k i n}\right)$ are the components (not to be confused with Cartesian components) of the kinetic spin-current density. Now, we use Eq. (8) together with Eqs. (9) and (10) to find

$$
\begin{aligned}
\vec{j}^{k i n}\left(\vec{r}, \vec{r}^{\prime}\right)= & \frac{\hbar}{2 m i} \frac{1}{2 \pi i} \int_{c-i \infty}^{c+i \infty} d \beta \frac{e^{\beta E_{F}}}{\beta}\left[\left(\vec{\nabla}_{\vec{r}}\right.\right. \\
& \left.\left.-\vec{\nabla}_{\vec{r}^{\prime}}\right) \sum_{\sigma} C_{\beta}\left(\vec{r}, \sigma ; \vec{r}^{\prime}, \sigma\right)\right],
\end{aligned}
$$




$$
\begin{aligned}
\vec{J}_{\lambda=1,2,3}^{k i n}\left(\vec{r}, \vec{r}^{\prime}\right)= & \frac{\hbar}{2 m i} \frac{1}{2 \pi i} \int_{c-i \infty}^{c+i \infty} d \beta \frac{e^{\beta E_{F}}}{\beta}\left[\left(\vec{\nabla}_{\vec{r}}\right.\right. \\
& \left.\left.-\vec{\nabla}_{\vec{r}^{\prime}}\right) \sum_{\sigma, \sigma^{\prime}} C_{\beta}\left(\vec{r}, \sigma ; \vec{r}^{\prime}, \sigma^{\prime}\right)\left\langle\sigma^{\prime}\left|\sigma_{\lambda}\right| \sigma\right\rangle\right] .
\end{aligned}
$$

It should be noted that when Hamiltonian of the system includes a spin-orbit coupling term, the corresponding particle current and spin current densities are no longer simply given by Eqs. (12) and (13) but contain additional terms. To obtain these terms, let us use the following definitions and physically reasonable Hermitian forms for the particle current density operator:

$$
\vec{j}=\frac{1}{2} \sum_{j=1}^{N}\left[\vec{v}_{j} \delta\left(\vec{r}-\vec{r}_{j}\right)+\delta\left(\vec{r}-\vec{r}_{j}\right) \vec{v}_{j}\right],
$$

and for the spin current density operator,

$$
\vec{J}_{\lambda=1,2,3}=\frac{1}{2} \sum_{j=1}^{N}\left[\vec{v}_{j}\left(\sigma_{j}\right)_{\lambda} \delta\left(\vec{r}-\vec{r}_{j}\right)+\delta\left(\vec{r}-\vec{r}_{j}\right)\left(\sigma_{j}\right)_{\lambda} \vec{v}_{j}\right],
$$

where $j$ labels the particles, and $\vec{p}_{j}$ and $\vec{v}_{j}=\left(\vec{\nabla}_{\vec{p}_{j}} \hat{H}_{0}\right)$ are, respectively, the momentum and the velocity operators of the particle $j$. Here, $\hat{H}_{0}$ is the corresponding many particle Hamiltonian and $\left(\sigma_{j}\right)_{\lambda}$ is the $2 \times 2$ Pauli matrix acting on the spin space of the particle $j$. In the presence of the spin-orbit coupling, the one particle hamiltonian contains an extra contribution, which can be written in the case of linear in momentum spin orbit term, as $\hat{H}_{s o}=\hbar\left(\sum_{\mu=1}^{3} \vec{A}_{\mu} \sigma_{\mu}\right) \cdot \vec{p},{ }^{22,23}$ where the set of the three vectors $\vec{A}_{1}, \vec{A}_{2}$, and $\vec{A}_{3}$ will be explicitly given further for the case of Rashba and/or Dresselhauss spin orbit coupling. In the absence of a magnetic field, we get $\vec{v}$ $=\frac{\vec{p}}{m}+\hbar \sum_{\nu=1}^{3} \vec{A}_{\nu} \sigma_{\nu}$ for the velocity operator, which yields to the following expressions for the one particle current and the spin current densities:

$$
\begin{gathered}
\vec{j}=\vec{j}^{k i n}+\hbar \sum_{\nu=1}^{3} \rho_{\nu} \vec{A}_{\nu}, \\
\vec{J}_{\lambda}=\vec{J}_{\lambda}^{k i n}+\hbar \rho \vec{A}_{\lambda}, \quad \lambda=1,2,3,
\end{gathered}
$$

where $\vec{j}^{k i n}$ and $\vec{J}_{\lambda}^{k i n}$ are, respectively, given in Eqs. (12) and (13) and $\rho_{\nu}$ is the $\nu$ th component of the spin vector density $\vec{\rho}$. Notice that the latter density and the spin current density $\vec{J}_{\lambda}$ are related by a continuity equation. Several authors have derived such relation [see Refs. 24-26 and references therein]. We can show, using our notations, that it reads

$$
\frac{\partial \rho_{\lambda}}{\partial t}+\frac{1}{2} \vec{\nabla} \cdot \vec{J}_{\lambda}=m \sum_{\mu=1}^{3} \sum_{\nu=1}^{3} \epsilon_{\lambda \mu \nu} \vec{A}_{\mu} \cdot \vec{J}_{\nu}, \quad \lambda=1,2,3 .
$$

Let us mention that in the context of time independent spin current density functional theory, a general relationship between the spin vector density $\vec{\rho}$ and the spin current density $\vec{J}_{\lambda}$ has been obtained earlier in Refs. 22 and 23, including a scalar potential, an external magnetic field, and spin orbit coupling. In the absence of magnetic field, the equation that is obtained there reduces to the static form of Eq. (18), i.e., without the term $\frac{\partial \rho_{\lambda}}{\partial t}$.

Hence, the various physical local densities of the system, namely, $\rho, \vec{\rho}, \vec{j}$, and $\vec{J}_{\lambda}$, have been expressed in terms of the Bloch density matrix $C_{\beta}\left(\vec{r}, \sigma ; \vec{r}^{\prime}, \sigma^{\prime}\right)$. Therefore, if one succeeds in finding an explicit analytical solution of the Bloch equation (3), then the previously mentioned local densities can be deduced without going through the use of the single particle wave functions.

Finally, some comments can be made about closed analytical solutions of Eq. (3) for simple but interesting physical situations. The interest in finding closed analytical expression for the Bloch density matrix or Bloch propagator is an old problem ${ }^{27}$ but is still of considerable interest. In the absence of the spin-orbit term in the Hamiltonian, exact analytical forms for the Bloch density matrix have already been derived in the case of an isotropic harmonic potential ${ }^{27}$ and also for a three- or two-dimensional charged isotropic oscillator in a uniform magnetic field. ${ }^{28}$ In recent years, these useful analytical results were used in theoretical studies of ultracold atoms. In fact, with the advances in the magnetooptical trapping techniques, it becomes possible to obtain harmonically confined ultracold atoms. ${ }^{29}$ These experimental realizations of a nearly noninteracting system have motivated a considerable volume of theoretical work ${ }^{30}$ and provide a natural ground for the application of the Bloch density matrix method at zero and nonzero temperatures.

Let us now come to the case where, in addition to a scalar potential, a spin-orbit term is also present. For such a spindependent Hamiltonian, to our knowledge, a closed analytical expression for the associated Bloch density matrix has not been reported in the literature yet, even for the simple case of Rashba or Dresselhaus spin-orbit coupling with a harmonic confining potential. In Sec. III, we shall consider the case of the Hamiltonian given by Eq. (1), which corresponds to situations in which the scalar potential and the Zeeman-like terms are set to zero in Eq. (5).

\section{EQUILIBRIUM PERSISTENT SPIN CURRENT IN TWO-DIMENSIONAL ELECTRON GAS}

For the 2DEG system under study, whose one particle Hamiltonian is given by Eq. (1), one can apply our general method outlined above. As stated before, this corresponds to the simple case, where in Eq. (5) $\hat{V}=\frac{\kappa}{\hbar}\left(P_{y} \sigma_{x}-P_{x} \sigma_{y}\right)$ $+\frac{\gamma}{\hbar}\left(P_{y} \sigma_{y}-P_{x} \sigma_{x}\right)$, i.e., we have no confining potential and we ignore the Zeeman-like term. By using the plane wave basis, Eq. (2) leads to

$$
\begin{aligned}
C_{\beta}\left(\vec{r}, \sigma ; \vec{r}^{\prime}, \sigma^{\prime}\right) & \left.=\langle\vec{r}, \sigma| e^{-\beta \cdot \vec{P}^{2} / 2 m} e^{-\beta \hat{V}}\right)\left|\vec{r}^{\prime}, \sigma^{\prime}\right\rangle \\
& =\int \frac{d^{2} p}{(2 \pi \hbar)^{2}} e^{(i / \hbar) \vec{p} \cdot\left(\vec{r}-\vec{r}^{\prime}\right)} e^{-\beta \vec{p}^{2} / 2 m}\left\langle\sigma\left|e^{-\beta|\vec{a}| \vec{u} \cdot \vec{\sigma}}\right| \sigma^{\prime}\right\rangle,
\end{aligned}
$$

where the components $u_{\lambda=1,2,3}$ of the unit vector $\vec{u}=\vec{a} /|\vec{a}|$ are given by 


$$
u_{1}=\frac{1}{\hbar}\left(\kappa p_{y}-\gamma p_{x}\right) /|\vec{a}|, \quad u_{2}=\frac{1}{\hbar}\left(-\kappa p_{x}+\gamma p_{y}\right) /|\vec{a}|, \quad u_{3}=0,
$$

with

$$
|\vec{a}|=\frac{1}{\hbar} \sqrt{\left(\kappa^{2}+\gamma^{2}\right)\left[p_{x}^{2}+p_{y}^{2}-4 \kappa \gamma p_{x} p_{y} /\left(\kappa^{2}+\gamma^{2}\right)\right]} .
$$

Next, we use the following expansion $e^{-\beta|\vec{a}| \vec{u} \cdot \vec{\sigma}}=\cosh (\beta|\vec{a}|)$ $-(\vec{u} \cdot \vec{\sigma}) \sinh (\beta|\vec{a}|)$, we can then write Eq. (19) as

$$
\begin{aligned}
C_{\beta}\left(\vec{r}, \sigma ; \vec{r}^{\prime}, \sigma^{\prime}\right)= & \int \frac{d^{2} p}{(2 \pi \hbar)^{2}} e^{(i / \hbar) \vec{p} \cdot\left(\vec{r}-\vec{r}^{\prime}\right)} e^{-\beta \vec{p}^{2} / 2 m} \cosh (\beta|\vec{a}|) \delta_{\sigma \sigma^{\prime}} \\
& -\int \frac{d^{2} p}{(2 \pi \hbar)^{2}} e^{(i / \hbar) \vec{p} \cdot\left(\vec{r}-\vec{r}^{\prime}\right)} e^{-\beta \vec{p}^{2} / 2 m} \\
& \times \sinh (\beta|\vec{a}|) \vec{u} \cdot\left\langle\sigma|\vec{\sigma}| \sigma^{\prime}\right\rangle .
\end{aligned}
$$

Once the Bloch density matrix is known, we proceed to evaluate the various local densities, $\rho, \vec{\rho}, \vec{j}$, and $\vec{J}_{\lambda}$ by the use of Eqs. (9), (10), (16), and (17) with Eqs. (12) and (13). Therefore, the substitution of Eq. (22) into (9) leads to

$$
\begin{aligned}
\rho(\vec{r}) & =\frac{2}{2 \pi i} \int_{c-i \infty}^{c+i \infty} \frac{d \beta}{\beta} e^{\beta E_{F}} \int e^{\left(-\beta \vec{p}^{2} / 2 m\right)} \cosh (\beta|\vec{a}|) \frac{d^{2} p}{(2 \pi \hbar)^{2}} \\
& =\int \frac{d^{2} p}{(2 \pi \hbar)^{2}}\left[\theta\left(E_{F}-\vec{p}^{2} / 2 m+|\vec{a}|\right)+\theta\left(E_{F}-\vec{p}^{2} / 2 m-|\vec{a}|\right)\right],
\end{aligned}
$$

where we have used the following relation:

$$
\begin{aligned}
& \frac{1}{2 \pi i} \int_{c-i \infty}^{c+i \infty} \frac{d \beta}{\beta} \exp \beta\left(E_{F}-\vec{p}^{2} / 2 m \pm|\vec{a}|\right) \\
& \quad=\theta\left(E_{F}-\vec{p}^{2} / 2 m \pm|\vec{a}|\right) .
\end{aligned}
$$

The $\vec{p}$ integration can be easily performed by using the polar coordinates of the momentum, $p_{x}=p \cos (\phi)$ and $p_{y}$ $=p \sin (\phi)$, simple manipulations lead to the following result:

$$
\rho(\vec{r})=\frac{1}{\pi \hbar^{2}}\left[m E_{F}+\frac{m^{2}}{\hbar^{2}}\left(\kappa^{2}+\gamma^{2}\right)\right] .
$$

Here, $E_{F}$ is the Fermi energy which could be expressed in terms of the total particle number $N$ through the normalization of the density. By putting again Eq. (22) into Eqs. (10) and (16), we find that the spin vector density and the particle current density vanish everywhere, that is,

$$
\vec{\rho}(\vec{r})=\overrightarrow{0} \text { and } \vec{j}(\vec{r})=\overrightarrow{0} .
$$

The above results in Eq. (26) could be found by using general arguments from symmetry considerations, as noticed in Ref. 26. In fact, the Hamiltonian in Eq. (1) commutes with the time reversal operator $T$; this leads to the Kramer degeneracy. The properties of the single particle density matrix $\rho\left(\vec{r}, \sigma ; \vec{r}, \sigma^{\prime}\right)$ under time reversal are carefully examined in Ref. 31, and, in particular, it is shown that if such a density matrix is invariant under time reversal, i.e., $t \rightarrow-t$ transfor- mation, then the local spin vector density $\vec{\rho}(\vec{r})$ and the local current density $\vec{j}(\vec{r})$ vanish.

Although the particle current density is identically zero, we show in the following that the spin current density is nonzero. This is due to the presence of the spin orbit term in the Hamiltonian. We first explicitly calculate the local kinetic spin current in Eq. (13). Substituting Eq. (22) into (13) and performing the inverse Laplace transform implies the following reduced expression for the local kinetic spin current density:

$$
\begin{aligned}
\vec{J}_{\lambda=1,2,3}^{k i n}(\vec{r})= & -\int \frac{d^{2} p}{(2 \pi \hbar)^{2}} \frac{\vec{p}}{m} u_{\lambda}\left[\theta\left(E_{F}-\vec{p}^{2} / 2 m+|\vec{a}|\right)-\theta\left(E_{F}\right.\right. \\
& \left.\left.-\vec{p}^{2} / 2 m-|\vec{a}|\right)\right] .
\end{aligned}
$$

By using Eq. (20) together with (21), we evaluate the above integral using polar coordinates in momentum space. This gives the following results for $\vec{J}_{1}^{k i n}$ and $\vec{J}_{2}^{k i n}$ with $\vec{J}_{3}^{k i n}=\overrightarrow{0}$ :

$$
\begin{aligned}
\vec{J}_{1}^{k i n}= & +\frac{\gamma m}{3 \pi \hbar^{3}}\left[\frac{2 m}{\hbar^{2}}\left(2 \kappa^{2}+\gamma^{2}\right)+3 E_{F}\right] \hat{x}-\frac{\kappa m}{3 \pi \hbar^{3}}\left[\frac { 2 m } { \hbar ^ { 2 } } \left(\kappa^{2}\right.\right. \\
& \left.\left.+2 \gamma^{2}\right)+3 E_{F}\right] \hat{y}, \\
\vec{J}_{2}^{k i n}= & +\frac{\kappa m}{3 \pi \hbar^{3}}\left[\frac{2 m}{\hbar^{2}}\left(\kappa^{2}+2 \gamma^{2}\right)+3 E_{F}\right] \hat{x}-\frac{\gamma m}{3 \pi \hbar^{3}}\left[\frac { 2 m } { \hbar ^ { 2 } } \left(2 \kappa^{2}\right.\right. \\
& \left.\left.+\gamma^{2}\right)+3 E_{F}\right] \hat{y} .
\end{aligned}
$$

According to Eq. (17), to obtain the spin current density, we need only to calculate the set of the three spin-orbit vectors $\vec{A}_{1}, \vec{A}_{2}$, and $\vec{A}_{3}$ since the kinetic spin current and the particle density are now known. Let us rewrite the spin orbit term in Eq. (1) as

$$
\frac{\kappa}{\hbar}\left(P_{y} \sigma_{x}-P_{x} \sigma_{y}\right)+\frac{\gamma}{\hbar}\left(P_{y} \sigma_{y}-P_{x} \sigma_{x}\right)=\hbar\left(\sum_{\mu=1}^{3} \vec{A}_{\mu} \sigma_{\mu}\right) \vec{P},
$$

where we recall that $\vec{P}$ is the momentum operator. We then deduce from Eq. (30) that

$$
\vec{A}_{1}=-\frac{\gamma}{\hbar^{2}} \hat{x}+\frac{\kappa}{\hbar^{2}} \hat{y}, \quad \vec{A}_{2}=-\frac{\kappa}{\hbar^{2}} \hat{x}+\frac{\gamma}{\hbar^{2}} \hat{y}, \quad \vec{A}_{3}=\overrightarrow{0} .
$$

Now, by substituting Eqs. (28), (29), (25), and (31) into Eq. (17), we then get the following expression for the spin current:

$$
\begin{aligned}
& \vec{J}_{1}=+\frac{m^{2} \gamma}{3 \pi \hbar^{5}}\left(\kappa^{2}-\gamma^{2}\right) \hat{x}+\frac{m^{2} \kappa}{3 \pi \hbar^{5}}\left(\kappa^{2}-\gamma^{2}\right) \hat{y} \\
& \vec{J}_{2}=-\frac{m^{2} \kappa}{3 \pi \hbar^{5}}\left(\kappa^{2}-\gamma^{2}\right) \hat{x}-\frac{m^{2} \gamma}{3 \pi \hbar^{5}}\left(\kappa^{2}-\gamma^{2}\right) \hat{y} .
\end{aligned}
$$

The above results are similar to those recently obtained in Ref. 8. 


\section{SPIN DENSITY MATRIX METHOD AND EQUILIBRIUM PERSISTENT SPIN CURRENT IN TWO- DIMENSIONAL ELECTRON GAS AT NONZERO TEMPERATURES}

In the following, we shall generalize the method and the results that are obtained in the previous section at $T=0$ to nonzero temperatures. To do so, we start with the important relation given in Eq. (6), which expresses the density operator in terms of the Bloch density. Such relation was generalized at finite temperature, ${ }^{32}$ it reads

$$
\hat{\rho}_{\mu}=\frac{1}{2 \pi i} \int_{c-i \infty}^{c+i \infty} d \beta e^{\beta \mu} \frac{\hat{C}_{\beta}^{T}}{\beta},
$$

where

$$
\hat{C}_{\beta}^{T}=\hat{C}_{\beta}^{T=0} \frac{\left(\pi \beta k_{B} T\right)}{\sin \left(\pi \beta k_{B} T\right)}
$$

is the finite temperature extension of the Bloch density operator, $\mu$ is the chemical potential, $k_{B}$ is Boltzmann's constant, and $\hat{C}_{\beta}^{T=0}=\hat{C}_{\beta}$ is the $T=0$ Bloch density operator given in Eq. (2). Recall that in the present context, $\beta$ is to be interpreted as mathematical variable, which in general is taken to be complex and not the inverse temperature $1 / k_{B} T$. For the interested reader, we give in the following a simple proof of Eq. (34). Using the grand canonical description for noninteracting fermions, the density operator at temperature $T$ is given by

$$
\hat{\rho}_{\mu}=\sum_{\text {all } i}\left|\Psi_{i}\right\rangle\left\langle\Psi_{i}\right| f_{i}
$$

Here, $f_{i}=\left[e^{\left(\epsilon_{i}-\mu\right) / k_{B} T}+1\right]^{-1}$ is the Fermi distribution function. If we use the identity

$$
\frac{1}{e^{x}+1}=\int_{-\infty}^{+\infty} \frac{\theta(y-x)}{4 \cosh ^{2}\left(\frac{y}{2}\right)} d y,
$$

we deduce that

$$
f_{i}=\int_{-\infty}^{+\infty} \frac{\theta\left(\varepsilon-\epsilon_{i}+\mu\right)}{4 k_{B} T \cosh ^{2}\left(\frac{\epsilon}{2 k_{B} T}\right)} d \epsilon .
$$

By writing the Heaviside step function as an inverse Laplace transform like in Eq. (7), the above relation becomes

$$
f_{i}=\frac{1}{2 \pi i} \int_{c-i \infty}^{c+i \infty} d \beta \frac{e^{\beta \mu}}{\beta} e^{-\beta \epsilon_{i}} \frac{\left(\pi \beta k_{B} T\right)}{\sin \left(\pi \beta k_{B} T\right)},
$$

where we have used the following two sided Laplace transform (see, e.g., Ref. 33):

$$
\int_{-\infty}^{+\infty} \frac{e^{-\beta \epsilon}}{4 k_{B} T \cosh ^{2}\left(\frac{\epsilon}{2 k_{B} T}\right)} d \epsilon=\frac{\left(\pi \beta k_{B} T\right)}{\sin \left(\pi \beta k_{B} T\right)} .
$$

By substituting Eq. (40) into Eq. (36), we obtain

$$
\begin{aligned}
\hat{\rho}_{\mu} & =\frac{1}{2 \pi i} \int_{c-i \infty}^{c+i \infty} d \beta \frac{e^{\beta \mu}}{\beta}\left(\sum_{\text {all } i}\left|\Psi_{i}\right\rangle\left\langle\Psi_{i}\right| e^{-\beta \epsilon_{i}}\right) \frac{\left(\pi \beta k_{B} T\right)}{\sin \left(\pi \beta k_{B} T\right)} \\
& =\frac{1}{2 \pi i} \int_{c-i \infty}^{c+i \infty} d \beta e^{\beta \mu} \frac{\exp (-\beta \hat{H})}{\beta} \frac{\left(\pi \beta k_{B} T\right)}{\sin \left(\pi \beta k_{B} T\right)} \\
& =\frac{1}{2 \pi i} \int_{c-i \infty}^{c+i \infty} d \beta e^{\beta \mu} \frac{\hat{C}_{\beta}^{T}}{\beta} .
\end{aligned}
$$

This completes the proof of the relation given in Eq. (34).

Notice that the finite temperature single particle density operator is expressed as an inverse Laplace transform in a similar form as in the $T=0$ case. Therefore, we can use the spin density matrix method that is previously developed to obtain the local densities $\rho_{T}, \vec{\rho}_{T}, \vec{j}_{T}$, and $\vec{J}_{T \lambda}$ at nonzero temperatures. This is simply achieved by including in Eqs. (9)-(13) with Eqs. (16) and (17) the temperature dependent factor $\left(\pi \beta k_{B} T\right) / \sin \left(\pi \beta k_{B} T\right)$ and replacing the Fermi energy $E_{F}$ by the chemical potential $\mu$. Consequently, it follows

$$
\begin{gathered}
\rho_{T}(\vec{r})=\frac{1}{2 \pi i} \int_{c-i \infty}^{c+i \infty} d \beta \frac{e^{\beta \mu}}{\beta}\left[\sum_{\sigma} C_{\beta}^{T=0}(\vec{r}, \sigma ; \vec{r}, \sigma) \frac{\left(\pi \beta k_{B} T\right)}{\sin \left(\pi \beta k_{B} T\right)}\right] \\
\vec{\rho}_{T}(\vec{r})= \\
\qquad \frac{1}{2 \pi i} \int_{c-i \infty}^{c+i \infty} d \beta \frac{e^{\beta \mu}}{\beta}\left[\sum_{\sigma, \sigma^{\prime}} C_{\beta}^{T=0}\left(\vec{r}, \sigma ; \vec{r}, \sigma^{\prime}\right)\right. \\
\left.\quad \times\left\langle\sigma^{\prime}|\vec{\sigma}| \sigma\right\rangle \frac{\left(\pi \beta k_{B} T\right)}{\sin \left(\pi \beta k_{B} T\right)}\right]
\end{gathered}
$$

and the finite temperature versions of Eqs. (16) and (17) are, respectively,

$$
\vec{j}_{T}=\vec{j}_{T}^{k i n}+\hbar \sum_{\nu=1}^{3} \rho_{T \nu} \vec{A}_{\nu}
$$

$$
\vec{J}_{T \lambda}=\vec{J}_{T \lambda}^{k i n}+\hbar \rho_{T} \vec{A}_{\lambda}, \quad \lambda=1,2,3,
$$

with

$$
\begin{aligned}
\vec{j}_{T}^{k i n}(\vec{r})= & \frac{\hbar}{2 m i} \frac{1}{2 \pi i} \int_{c-i \infty}^{c+i \infty} d \beta \frac{e^{\beta \mu}}{\beta}\left[\left(\vec{\nabla}_{\vec{r}}\right.\right. \\
& \left.\left.-\vec{\nabla}_{\vec{r}^{\prime}}\right) \sum_{\sigma} C_{\beta}^{T=0}\left(\vec{r}, \sigma ; \vec{r}^{\prime}, \sigma\right) \frac{\left(\pi \beta k_{B} T\right)}{\sin \left(\pi \beta k_{B} T\right)}\right]_{\vec{r}^{\prime}=\vec{r}},
\end{aligned}
$$




$$
\begin{aligned}
\vec{J}_{T \lambda=1,2,3}^{k i n}(\vec{r})= & \frac{\hbar}{2 m i} \frac{1}{2 \pi i} \int_{c-i \infty}^{c+i \infty} d \beta \frac{e^{\beta \mu}}{\beta}\left[\left(\vec{\nabla}_{\vec{r}}\right.\right. \\
& \left.-\vec{\nabla}_{\vec{r}^{\prime}}\right) \sum_{\sigma, \sigma^{\prime}} C_{\beta}^{T=0}\left(\vec{r}, \sigma ; \vec{r}^{\prime}, \sigma^{\prime}\right) \\
& \left.\times\left\langle\sigma^{\prime}\left|\sigma_{\lambda}\right| \sigma\right\rangle \frac{\left(\pi \beta k_{B} T\right)}{\sin \left(\pi \beta k_{B} T\right)}\right]_{\vec{r}^{\prime}=\vec{r}},
\end{aligned}
$$

where we recall that $C_{\beta}^{T=0}\left(\vec{r}, \sigma ; \vec{r}^{\prime}, \sigma^{\prime}\right)$ is the Bloch density matrix at $T=0$. In the above, for notational simplicity, we have only displayed the local parts of the various densities. Hence, in a similar way as in the $T=0$ case, the actual densities $\rho_{T}, \vec{\rho}_{T}, \vec{j}_{T}$, and $\vec{J}_{T \lambda}$ have been expressed in terms of
$C_{\beta}^{T=0}$, which in turn can be obtained through the solution of the Bloch equation (3).

In the following, we shall apply the spin density matrix method at finite temperature to the unconfined 2DEG system examined in the previous section at $T=0$. For that, we only need to insert the expression of the "cold Bloch density" $C_{\beta}^{T=0}\left(\vec{r}, \sigma ; \vec{r}^{\prime}, \sigma^{\prime}\right)$, which is given in Eq. (22) into Eq. (41), to get the particle density for a given temperature $T$,

$$
\begin{aligned}
\rho_{T}= & \int \frac{d^{2} p}{(2 \pi \hbar)^{2}} \frac{1}{2 \pi i} \int_{c-i \infty}^{c+i \infty} \frac{d \beta}{\beta} e^{\beta \mu} e^{\left(-\beta \vec{p}^{2} / 2 m\right)}\left[e^{\beta|\vec{a}|}\right. \\
& \left.+e^{-\beta|\vec{a}|}\right] \frac{\left(\pi \beta k_{B} T\right)}{\sin \left(\pi \beta k_{B} T\right)} .
\end{aligned}
$$

To carry out the inverse Laplace transform, we make use of Eq. (40) to obtain

$$
\begin{aligned}
\rho_{T} & =\int \frac{d^{2} p}{(2 \pi \hbar)^{2}} \int_{-\infty}^{+\infty} \frac{d \epsilon}{4 k_{B} T \cosh ^{2}\left(\frac{\epsilon}{2 k_{B} T}\right)} \int_{c-i \infty}^{c+i \infty} \frac{e^{\beta\left(\mu-\vec{p}^{2} / 2 m+|\vec{a}|-\epsilon\right)}+e^{\beta\left(\mu-\vec{p}^{2} / 2 m-|\vec{a}|-\epsilon\right)}}{2 \pi i \beta} d \beta \\
& =\int \frac{d^{2} p}{(2 \pi \hbar)^{2}} \int_{-\infty}^{+\infty} \frac{\left[\theta\left(\mu-\vec{p}^{2} / 2 m+|\vec{a}|-\epsilon\right)+\theta\left(\mu-\vec{p}^{2} / 2 m-|\vec{a}|-\epsilon\right)\right]}{4 k_{B} T \cosh ^{2}\left(\frac{\epsilon}{2 k_{B} T}\right)} d \epsilon \\
& =\int \frac{d^{2} p}{(2 \pi \hbar)^{2}}\left[\frac{1}{\exp \left(\frac{\vec{p}^{2} / 2 m-|\vec{a}|-\mu}{k_{B} T}\right)+1}+\frac{\exp \left(\frac{\vec{p}^{2} / 2 m+|\vec{a}|-\mu}{k_{B} T}\right)+1}{1}\right] .
\end{aligned}
$$

The above expression is an exact quantum mechanical result. Note that it looks as if we have simply replaced by hand in Eq. (23), the step function by the Fermi distribution function. Taking into account this last remark, one is easily convinced that the spin vector density $\vec{\rho}_{T}(\vec{r})$ and the current density $\vec{j}_{T}(\vec{r})$ vanish as in the $T=0$ case. Moreover, by using Eq. (27), we deduce the finite temperature expression of the kinetic spin current density as

$$
\begin{aligned}
\vec{J}_{T \lambda=1,2,3}^{k i n}(\vec{r})= & -\int \frac{d^{2} p}{(2 \pi \hbar)^{2}} \frac{\vec{p}}{m} u_{\lambda}\left[\frac{1}{\exp \left(\frac{\vec{p}^{2} / 2 m-|\vec{a}|-\mu}{k_{B} T}\right)+1}\right. \\
& \left.-\frac{1}{\exp \left(\frac{\vec{p}^{2} / 2 m+|\vec{a}|-\mu}{k_{B} T}\right)+1}\right] .
\end{aligned}
$$

To push the analytical treatment as far as possible, we shall, in the remaining of the paper, restrict ourselves to the case where only the Rashba spin-orbit coupling is present. Let us mention that the case of pure Dresslhauss spin orbit coupling can also be analytically worked out along the same way, as will be described in the following. Therefore, by putting $\gamma=0$, Eq. (21) gives

$$
|\vec{a}|=\frac{\kappa}{\hbar}|\vec{p}|
$$

so that, carrying out the angular integration, the particle density in Eq. (48) simplifies to

$$
\begin{aligned}
\rho_{T}= & \frac{1}{2 \pi \hbar^{2}} \int_{0}^{+\infty} d p\left[\frac{p}{\exp \left(\frac{p^{2} / 2 m-\frac{\kappa}{\hbar} p-\mu}{k_{B} T}\right)+1}\right. \\
& \left.+\frac{p}{\exp \left(\frac{p^{2} / 2 m+\frac{\kappa}{\hbar} p-\mu}{k_{B} T}\right)+1}\right]
\end{aligned}
$$

which can be rewritten as 


$$
\begin{aligned}
& \rho_{T}=\frac{m}{2 \pi \hbar^{2}} \int_{0}^{+\infty} d p\left[\frac{\frac{p}{m}-\frac{\kappa}{\hbar}}{\exp \left(\frac{p^{2} / 2 m-\frac{\kappa}{\hbar} p-\mu}{k_{B} T}\right)+1 \exp \left(\frac{\left.p^{2} / 2 m+\frac{\kappa}{\hbar} p-\mu\right)}{k_{B} T}\right)+1}\right] \\
& +\frac{m \kappa}{2 \pi \hbar^{3}} \int_{0}^{+\infty} d p\left[\frac{1}{\exp \left(\frac{p^{2} / 2 m-\frac{\kappa}{\hbar} p-\mu}{k_{B} T}\right)+1}-\frac{1}{\exp \left(\frac{p^{2} / 2 m+\frac{\kappa}{\hbar} p-\mu}{k_{B} T}\right)+1}\right] \\
& =\frac{m k_{B} T}{\pi \hbar^{2}} \ln \left(1+e^{\mu / k_{B} T}\right)+\frac{m \kappa}{2 \pi \hbar^{3}} \int_{0}^{+\infty} d p\left[\frac{1}{\exp \left(\frac{p^{2} / 2 m-\frac{\kappa}{\hbar} p-\mu}{k_{B} T}\right)+1}-\frac{1}{\exp \left(\frac{p^{2} / 2 m+\frac{\kappa}{\hbar} p-\mu}{k_{B} T}\right)+1}\right] .
\end{aligned}
$$

At this level, a perturbative treatment of the spin orbit is helpful. For that, let $g(\kappa)$ denotes the following function of the parameter $\kappa$ :

$$
\begin{aligned}
g(\kappa)= & \int_{0}^{+\infty} d p\left[\frac{1}{\exp \left(\frac{p^{2} / 2 m-\frac{\kappa}{\hbar} p-\mu}{k_{B} T}\right)+1}\right] \\
& \left.-\frac{1}{\exp \left(\frac{p^{2} / 2 m+\frac{\kappa}{\hbar} p-\mu}{k_{B} T}\right)+1}\right]
\end{aligned}
$$

Since $g(\kappa)$ is an odd function, its Taylor expansion around $\kappa=0$ up to the order $\kappa$ is $g(\kappa) \simeq \kappa(\partial g / \partial \kappa)_{\kappa=0}+O\left(\kappa^{3}\right)$, simple manipulations yield to

$$
g(\kappa)=\frac{(2 m \kappa) / \hbar}{1+e^{-\mu / k_{B} T}}+O\left(\kappa^{3}\right) .
$$

By substituting this result into Eq. (52), one obtains up to the order $\kappa^{2}$,

$$
\rho_{T}=\frac{m k_{B} T}{\pi \hbar^{2}} \ln \left(1+e^{\mu / k_{B} T}\right)+\frac{m^{2} \kappa^{2}}{\pi \hbar^{4}} \frac{1}{1+e^{-\mu / k_{B} T}} .
$$

The above equation is used to obtain the chemical potential $\mu$ for a given temperature $T$.

Let us now come to the evaluation of the kinetic spin current given in Eq. (49). Since $\gamma=0$, the components of the unit vector $\vec{u}$ in Eq. (20) reduce to $u_{1}=p_{y} / p$ and $u_{2}=-p_{x} / p$, with $u_{3}=0$. It then follows

$$
\begin{aligned}
\vec{J}_{T 1}^{k i n}= & -\int \frac{d^{2} p}{(2 \pi \hbar)^{2}} \frac{p_{y}^{2}}{m p}\left[\frac{1}{\exp \left(\frac{\vec{p}^{2} / 2 m-\frac{\kappa}{\hbar} p-\mu}{k_{B} T}\right)+1}\right. \\
& \left.-\frac{1}{\exp \left(\frac{\vec{p}^{2} / 2 m+\frac{\kappa}{\hbar} p-\mu}{k_{B} T}\right)+1}\right] \hat{y}=-\frac{1}{4 \pi m \hbar^{2}} h(\kappa) \hat{y},
\end{aligned}
$$

$$
\begin{aligned}
\vec{J}_{T 2}^{k i n}= & +\int \frac{d^{2} p}{(2 \pi \hbar)^{2}} \frac{p_{x}^{2}}{m p}\left[\frac{1}{\exp \left(\frac{\vec{p}^{2} / 2 m-\frac{\kappa}{\hbar} p-\mu}{k_{B} T}\right)+1}\right. \\
& \left.-\frac{1}{\exp \left(\frac{\vec{p}^{2} / 2 m+\frac{\kappa}{\hbar} p-\mu}{k_{B} T}\right)+1}\right] \hat{x}=\frac{1}{4 \pi m \hbar^{2}} h(\kappa) \hat{x}
\end{aligned}
$$

where in Eqs. (56) and (57) we have performed the angular integration and defined the function $h(\kappa)$ as 


$$
\begin{aligned}
h(\kappa)= & \int_{0}^{+\infty} d p\left[\frac{p^{2}}{\exp \left(\frac{p^{2} / 2 m-\frac{\kappa}{\hbar} p-\mu}{k_{B} T}\right)+1}\right] \\
& \left.-\frac{p^{2}}{\exp \left(\frac{p^{2} / 2 m+\frac{\kappa}{\hbar} p-\mu}{k_{B} T}\right)+1}\right]
\end{aligned}
$$

Expanding such odd function up to $\kappa^{3}$ order

$$
h(\kappa) \simeq \kappa\left(\frac{\partial h}{\partial \kappa}\right)_{\kappa=0}+\frac{\kappa^{3}}{6}\left(\frac{\partial^{3} h}{\partial \kappa^{3}}\right)_{\kappa=0}+O\left(\kappa^{5}\right) .
$$

Then, we substitute the following result, which is derived in the appendix:

$$
h(\kappa)=\frac{4 m^{2} \kappa\left(k_{B} T\right)}{\hbar} \ln \left(1+e^{\mu / k_{B} T}\right)+\frac{8 m^{3} \kappa^{3}}{3 \hbar^{3}} \frac{1}{1+e^{-\mu / k_{B} T}}+O\left(\kappa^{5}\right)
$$

into Eqs. (56) and (57) so that

$$
\begin{aligned}
\vec{J}_{T 1}^{k i n}= & -\left[\frac{m \kappa\left(k_{B} T\right)}{\pi \hbar^{3}} \ln \left(1+e^{\mu / k_{B} T}\right)+\frac{2 m^{2} \kappa^{3}}{3 \pi \hbar^{5}} \frac{1}{1+e^{-\mu / k_{B} T}}\right. \\
& \left.+O\left(\kappa^{5}\right)\right] \hat{y}, \\
\vec{J}_{T 2}^{k i n}= & +\left[\frac{m \kappa\left(k_{B} T\right)}{\pi \hbar^{3}} \ln \left(1+e^{\mu / k_{B} T}\right)+\frac{2 m^{2} \kappa^{3}}{3 \pi \hbar^{5}} \frac{1}{1+e^{-\mu / k_{B} T}}\right. \\
& \left.+O\left(\kappa^{5}\right)\right] \hat{x} .
\end{aligned}
$$

To determine the spin current, we make use of Eq. (44). By substituting into this equation the above two results [Eqs. (61) and (62)] together with Eqs. (55) and (31) for $\gamma=0$, one finally gets

$$
\begin{aligned}
& \vec{J}_{T 1}=+\left[\frac{m^{2} \kappa^{3}}{3 \pi \hbar^{5}} \frac{1}{1+e^{-\mu / k_{B} T}}+O\left(\kappa^{5}\right)\right] \hat{y}, \\
& \vec{J}_{T 2}=-\left[\frac{m^{2} \kappa^{3}}{3 \pi \hbar^{5}} \frac{1}{1+e^{-\mu / k_{B} T}}+O\left(\kappa^{5}\right)\right] \hat{x} .
\end{aligned}
$$

It is easy to check that, for $T \rightarrow 0$, the above expressions reduce, respectively, to their zero temperature limits given in Eqs. (32) and (33) with $\gamma=0$. This allows us to rewrite Eqs. (63) and (64), up to order $\kappa^{3}$, in the compact form

$$
\vec{J}_{T \lambda}=\vec{J}_{0 \lambda}\left(\frac{1}{1+e^{-\mu / k_{B} T}}\right), \quad \lambda=1,2,
$$

where obvious notations have been used. The above result is the generalization to nonzero temperatures of the one found in Ref. 18.

Next, to obtain the dependence of the spin current as a function of temperature $T$, one has to eliminate the chemical potential $\mu$ between Eqs. (55) and (65). To keep the accuracy

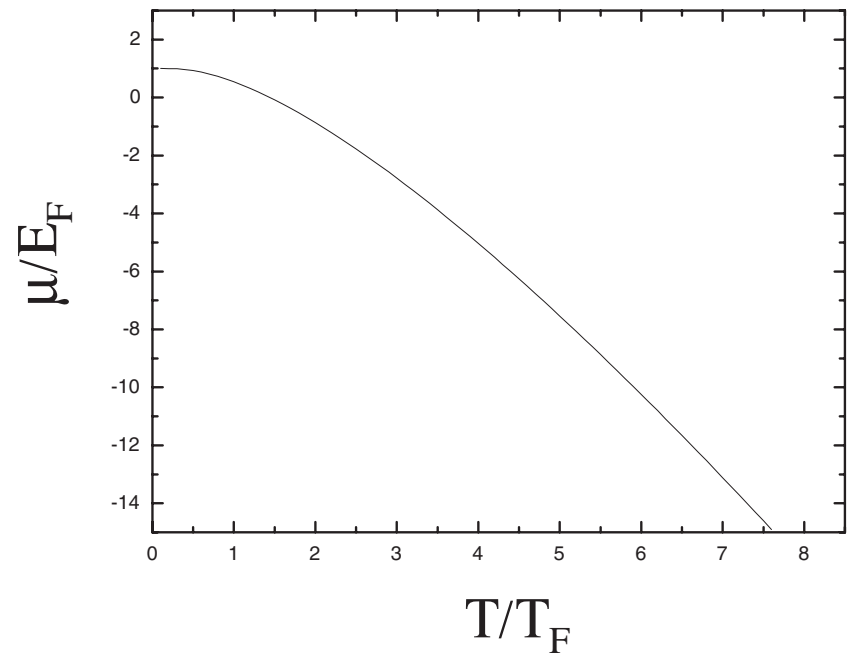
(68)].

FIG. 1. Plot of the ratio $\mu / E_{F}$ as a function of $T / T_{F}$ [see Eq.

consistently to order $\kappa^{3}$ in Eq. (65), we need to take only the contribution of the first term (leading term which is of order $\left.\kappa^{0}\right)$ in Eq. (55). So, at this order and for a given constant particle density $\rho$, this equation solves as

$$
\mu=k_{B} T \ln \left(e^{\pi \hbar^{2} \rho / m k_{B} T}-1\right) .
$$

At zero temperature, the above equation leads to the usual relation between the Fermi energy and the particle density in 2DEG, $E_{F}=\pi \hbar^{2} \rho / m$. At this point, let us introduce the Fermi temperature of the system $T_{F}=E_{F} / k_{B}$ so that

$$
T_{F}=\frac{\pi \hbar^{2} \rho}{m k_{B}} \text {. }
$$

Plugging this last relation into Eq. (66) yields

$$
\mu=E_{F} \frac{T}{T_{F}} \ln \left(e^{T_{F} / T}-1\right) .
$$

In Fig. 1, we plot the ratio $\mu / E_{F}$ as a function of $T / T_{F}$. Now, if we insert Eq. (68) into Eq. (65), one then finds

$$
\vec{J}_{T \lambda}=\vec{J}_{0 \lambda}\left[1-\exp \left(-\frac{T_{F}}{T}\right)\right] \text {. }
$$

In Fig. 2, the ratio $J_{T} / J_{0}$ is plotted as a function of $T / T_{F}$.

\section{SUMMARY AND OUTLOOK}

In this paper, we have presented a spin density matrix method at zero and nonzero temperatures for spin orbit coupled systems, using as a tool the Bloch density matrix. This density matrix is defined in a joint space of spin and orbital degrees of freedom. This allows us to calculate, beside the particle and spin densities, quantities of great interest such as the current and spin current. As a simple application, we have examined the case of unconfined 2DEG with Rashba spin-orbit coupling. We have found that the persistent spin current is extremely robust against thermal disorder. The results of these calculations can in principle be tested 


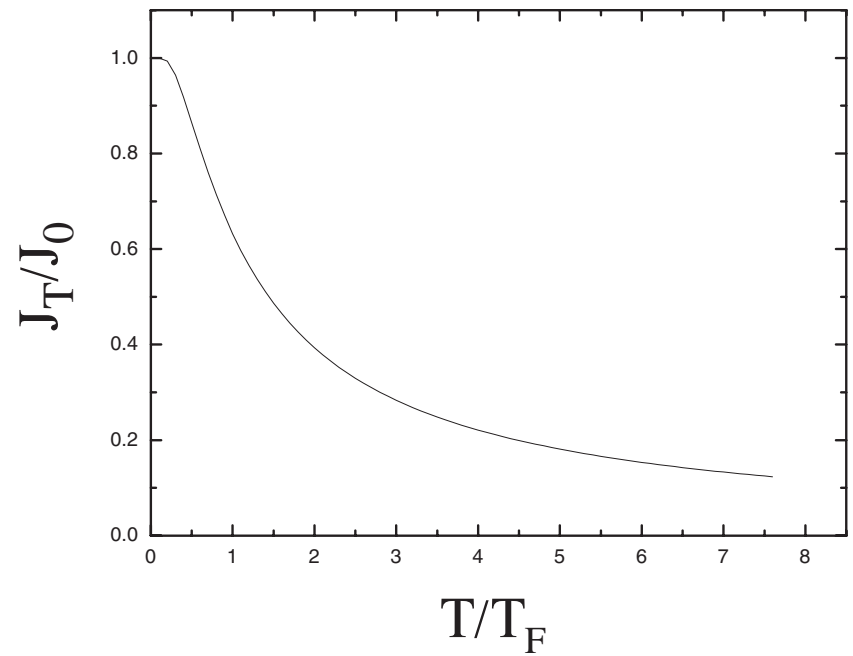

FIG. 2. Plot of the ratio $J_{T} / J_{0}$ as a function of $T / T_{F}$ [see Eq. (69)].

against mechanical torque experiments of the type recently suggested by Sonin. ${ }^{13}$ Natural extensions of the present study will be the inclusion of the effect of a confining scalar potential and the inclusion of many-body effects.

\section{ACKNOWLEDGMENT}

Thanks are due to A. Layadi for his help.

\section{APPENDIX A}

This appendix is devoted to the derivation of the result given in Eq. (60). To this aim, we start with Eq. (58), from which we deduce

$$
\kappa\left(\frac{\partial h}{\partial \kappa}\right)_{\kappa=0}=\frac{2 \kappa}{\hbar\left(k_{B} T\right)} \int_{0}^{+\infty} \frac{p^{3} A d p}{(1+A)^{2}}
$$

with

$$
A=\exp \left(\frac{p^{2} / 2 m-\mu}{k_{B} T}\right) .
$$

An integration by parts yields

$$
\kappa\left(\frac{\partial h}{\partial \kappa}\right)_{\kappa=0}=\frac{4 m^{2} \kappa\left(k_{B} T\right)}{\hbar} \ln \left(1+e^{\mu / k_{B} T}\right) .
$$

Using once again Eq. (58), we can show that

$$
\frac{\kappa^{3}}{6}\left(\frac{\partial^{3} h}{\partial \kappa^{3}}\right)_{\kappa=0}=\frac{\kappa^{3}}{3 \hbar^{3}\left(k_{B} T\right)^{3}} R,
$$

with

$$
\begin{aligned}
R & =\int_{0}^{+\infty} \frac{p^{5} A\left(A^{2}-4 A+1\right) d p}{(1+A)^{4}} \\
& =\int_{0}^{+\infty} \frac{p^{5} A d p}{(1+A)^{2}}-6 \int_{0}^{+\infty} \frac{p^{5} A^{2} d p}{(1+A)^{4}} .
\end{aligned}
$$

The first integral in Eq. (A5) can be carried out. An integration by parts yields

$$
\int_{0}^{+\infty} \frac{p^{5} A d p}{(1+A)^{2}}=4 m\left(k_{B} T\right) \int_{0}^{+\infty} \frac{p^{3} d p}{1+\exp \left(\frac{p^{2} / 2 m-\mu}{k_{B} T}\right)}
$$

If we make the change of variable $x=p^{2} /\left(2 m k_{B} T\right)$, we then get

$$
\int_{0}^{+\infty} \frac{p^{5} A d p}{(1+A)^{2}}=8 m^{3}\left(k_{B} T\right)^{3} F_{1}\left(\frac{\mu}{k_{B} T}\right)
$$

where $F_{1}$ is the so-called Fermi integral defined by

$$
F_{1}(z)=\int_{0}^{+\infty} \frac{x d x}{1+\exp (x-z)} .
$$

The second integral in Eq. (A5) can be performed as follows. We begin by two integration by parts

$$
\begin{aligned}
-6 \int_{0}^{+\infty} \frac{p^{5} A^{2} d p}{(1+A)^{4}}= & -2 m k_{B} T\left[4 \int_{0}^{+\infty} \frac{p^{3} A d p}{(1+A)^{3}}\right. \\
& \left.+\frac{k_{B} T}{m} \int_{0}^{+\infty} \frac{p^{5} A d p}{(1+A)^{3}}\right] \\
= & -2 m k_{B} T\left[4 m k_{B} T \int_{0}^{+\infty} \frac{p d p}{(1+A)^{2}}\right. \\
& \left.+2 \int_{0}^{+\infty} \frac{p^{3} d p}{(1+A)^{2}}\right] \\
= & -8 m^{3}\left(k_{B} T\right)^{3} \int_{0}^{+\infty}\left[1+\exp \left(x-\frac{\mu}{k_{B} T}\right)\right]^{2}
\end{aligned}
$$

Upon using

$$
\int_{0}^{+\infty} \frac{(x+1) d x}{\left.1+\exp \left(x-\frac{\mu}{k_{B} T}\right)\right]^{2}}=-\frac{1}{1+e^{-\mu / k_{B} T}}+F_{1}\left(\frac{\mu}{k_{B} T}\right)
$$

we find

$$
-6 \int_{0}^{+\infty} \frac{p^{5} A^{2} d p}{(1+A)^{4}}=8 m^{3}\left(k_{B} T\right)^{3}\left[\frac{1}{1+e^{-\mu / k_{B} T}}-F_{1}\left(\frac{\mu}{k_{B} T}\right)\right] .
$$

Summing up the two terms in Eq. (A5) that are given, respectively, by Eqs. (A7) and (A10), one then finds

$$
R=\frac{8 m^{3}\left(k_{B} T\right)^{3}}{1+e^{-\mu / k_{B} T}},
$$

thus, Eq. (A4) reads

$$
\frac{\kappa^{3}}{6}\left(\frac{\partial^{3} h}{\partial \kappa^{3}}\right)_{\kappa=0}=\frac{8 m^{3} \kappa^{3}}{3 \hbar^{3}}\left[\frac{1}{1+e^{-\mu / k_{B} T}}\right] .
$$

Finally, inserting Eqs. (A3) and (A12) into Eq. (59) will yield the result given in Eq. (60). 
${ }^{1}$ S. A. Wolf, D. D. Awschalom, R. A. Buhrman, J. M. Daughton, S. von Molnar, M. L. Roukes, A. Y. Chtchelkanova, and D. M. Treger, Science 294, 1488 (2001); I. Zutic, J. Fabian, and S. Das Sarma, Rev. Mod. Phys. 76, 323 (2004).

${ }^{2}$ Semiconductor Spintronics and Quantum Computation, edited by D. D. Awschalom, D. Loss, and N. Samarth (Springer, Berlin, 2002).

${ }^{3}$ E. I. Rashba, Physica E (Amsterdam) 20, 189 (2004).

${ }^{4}$ S. Datta and B. Das, Appl. Phys. Lett. 56, 665 (1990).

${ }^{5}$ J. Nitta, T. Akazaki, H. Takayanagi, and T. Enoki, Phys. Rev. Lett. 78, 1335 (1997).

${ }^{6}$ S. Murakami, N. Nagaosa, and S. C. Zhang, Science 301, 1348 (2003).

${ }^{7}$ J. Sinova, D. Culcer, Q. Niu, N. A. Sinitsyn, T. Jungwirth, and A. H. MacDonald, Phys. Rev. Lett. 92, 126603 (2004).

${ }^{8}$ J. Schliemann and D. Loss, Phys. Rev. B 71, 085308 (2005).

${ }^{9}$ J. Wunderlich, B. Kaestner, J. Sinova, and T. Jungwirth, Phys. Rev. Lett. 94, 047204 (2005).

${ }^{10}$ Y. K. Kato, R. C. Myers, A. C. Gossard, and D. D. Awschalom, Science 306, 1910 (2004).

${ }^{11}$ S. Murakami, Adv. Solid State Phys. 45, 197 (2005); J. Sinova, S. Murakami, S.-Q. Shen, and M.-S. Choi, Solid State Commun. 138, 214 (2006).

${ }^{12}$ E. M. Hankiewicz, G. Vignale, and M. E. Flatté, Phys. Rev. Lett. 97, 266601 (2006).

${ }^{13}$ E. B. Sonin, Phys. Rev. Lett. 99, 266602 (2007).

${ }^{14}$ E. B. Sonin, Phys. Rev. B 76, 033306 (2007).

${ }^{15}$ Y. A. Bychkov and E. I. Rashba, J. Phys. C 17, 6039 (1984).

${ }^{16}$ G. Dresselhaus, Phys. Rev. 100, 580 (1955).

${ }^{17}$ M. I. Dyakonov and V. Y. Kachorovskii, Sov. Phys. Semicond. 20, 110 (1986).

${ }^{18}$ E. I. Rashba, Phys. Rev. B 68, 241315(R) (2003).

${ }^{19}$ N. N. Lathiotakis, N. Helbig, and E. K. U. Gross, Phys. Rev. B 75, 195120 (2007).

${ }^{20}$ N. H. March and A. M. Murray, Phys. Rev. 120, 830 (1960).
${ }^{21}$ R. G. Parr and W. Yang, Density Functional Theory of Atoms and Molecules (Oxford Science, New York, 1989).

${ }^{22}$ G. Vignale and M. Rasolt, Phys. Rev. B 37, 10685 (1988).

${ }^{23}$ K. Bencheikh, J. Phys. A 36, 11929 (2003).

${ }^{24}$ A. A. Burkov, Alvaro S. Núñez, and A. H. MacDonald, Phys. Rev. B 70, 155308 (2004).

${ }^{25}$ S. I. Erlingsson, J. Schliemann, and D. Loss, Phys. Rev. B 71, 035319 (2005).

${ }^{26}$ Q. F. Sun and X. C. Xie, Phys. Rev. B 72, 245305 (2005); Y. Wang, K. Xia, Z. B. Su, and Z. Ma, Phys. Rev. Lett. 96, 066601 (2006).

${ }^{27}$ See, for instance, M. Hillery, R. F. O'Connel, M. O. Scully, and E. P. Wigner, Phys. Rep. 106, 121 (1984).

${ }^{28}$ N. H. March and M. P. Tosi, J. Phys. A 18, L643 (1985).

${ }^{29}$ B. De Marco B and D. S. Jin, Science 285, 1703 (1999); A. G. Truscott, K. E. Strecker, W. I. McAlexander, G. B. Partridge, and R. G. Hulet, Science 291, 2570 (2001); F. Schreck, L. Khaykovich, K. L. Corwin, G. Ferrari, T. Bourdel, J. Cubizolles, and C. Salomon, Phys. Rev. Lett. 87, 080403 (2001); S. R. Granade, M. E. Gehm, K. M. OHara, and J. E. Thomas, ibid. 88, 120405 (2002); G. Roati, F. Riboli, G. Modugno, and M. Inguscio, ibid. 89, 150403 (2002); Z. Hadzibabic, S. Gupta, C. A. Stan, C. H. Schunck, M. W. Zwierlein, K. Dieckmann, and W. Ketterle, ibid. 91, 160401 (2003).

${ }^{30}$ M. Brack and B. P. Van Zyl, Phys. Rev. Lett. 86, 1574 (2001); M. Brack M and M. V. N. Murthy, J. Phys. A 36, 1111 (2003); B. P. Van Zyl, R. K. Bhaduri, A. Suzuki, and M. Brack, Phys. Rev. A 67, 023609 (2003); P. Shea, and B. P. van Zyl, Phys. Rev. B 74, 205334 (2006); P. Shea and B. van Zyl, J. Phys. A 40, 10589 (2007).

${ }^{31}$ Y. M. Engel, D. M. Brink, K. Goeke, S. J. Krieger, and D. Vautherin, Nucl. Phys. A 249, 215 (1975).

${ }^{32}$ M. Brack, P. Quentin, Nucl. Phys. A 361, 35 (1981).

${ }^{33}$ B. van der Pohl and H. Bremmer, Operational Calculus, 2nd ed. (Cambridge University Press, Cambridge, UK, 1955). 